\title{
Pressão do cuff em pacientes em ventilação mecânica - relação com a mudança de decúbito
}

\author{
Cuff pressure in patients on mechanical ventilation - relationship with decubitus change
}

\author{
Elenice Gomes Ferreira ${ }^{a}$, Poliana Ricklib, Ênio Teixeira Molina Filhoc, Jefferson Fischerc, \\ Mateus Dias Antunes ${ }^{b}$, José Roberto Andrade do Nascimento Junior ${ }^{d}$, Daniel Vicentini de Oliveirae \\ a Fisioterapeuta. Mestre em Ciências da Saúde - Centro Universitário Cesumar (UNICESUMAR). \\ b Fisioterapeuta. UNICESUMAR. \\ c Médico. Hospital Metropolitano de Sarandi, Paraná. \\ d Educador Físico. Doutor em Educação Física - Universidade Estadual de Maringá (UEM). Professor dos Programas de Pós-Graduação em Educação Física e Psicologia \\ da Universidade Federal do Vale do São Francisco. \\ e Fisioterapeuta e Educador Físico. Mestre em Promoção da Saúde. Doutorando em Gerontologia - Universidade Estadual de Campinas (UNICAMP).
}

RESUMO

Objetivo: Verificar a pressão intra-cuff de pacientes sob ventilação mecânica e a relação com a mudança de decúbito. Materiais e Métodos: Trata-se de um estudo transversal. Para a verificação da pressão intra-cuff foi adaptado um cufômetro através de um manômetro, tendo como referência a pressão de $20 \mathrm{mmHg}$. No primeiro dia, inicialmente foi mensurada a pressão intra-cuff com o paciente em decúbito dorsal com cabeceira a 30ㅡ, seguido do decúbito lateral de costa para o ventilador e na sequencia o decúbito lateral de frente ao ventilador. Do segundo ao quinto dia foi verificada a pressão somente em decúbito dorsal com cabeceira a 30 ․ Para a análise dos dados foram utilizados os testes de Kolmogorov-smirnov e os testes $t$ de Student independente e dependente $(p<0,05)$.

Resultados: Nos cinco dias consecutivos de verificação da pressão intra-cuff na posição de decúbito dorsal observou-se que estas estavam levemente diminuídas comparadas ao valor de referência, onde o terceiro dia foi verificado a menor pressão $(16,4 \pm 15,1)$. Ao realizar a mudança de decúbito notou-se que as pressões intra-cuff apresentaram variações acima ou abaixo de $20 \mathrm{mmHg}$, que ao analisar a diferença entre as médias das pressões foram estatisticamente significativas $(p=0,001)$ e $(p=0,014)$. O decúbito lateral de costa para o ventilador apresentou-se com valores pressóricos dentro do referencial $(20,3 \pm \mathbf{6 , 5})$, enquanto houve diminuição no decúbito de frente para o ventilador $(18,1 \pm 4,8)$

Conclusão: Conclui-se que as médias das pressões intra-cuff em cinco dias consecutivos apresentaram-se diminuídas. Apenas no quinto dia as pressões mantiveram-se em valores considerados normais e a mudança de decúbito influenciou significativamente na alteração desta pressão.

Palavras-chave: serviço hospitalar de fisioterapia; unidades de terapia intensiva; respiração artificial.

Objective: To check the intra-cuff pressure of patients on mechanical ventilation and the relationship with the position change.

Materiais and Methods: This is a cross-sectional study. To check the cuff pressure, a manometer has been adjusted, with reference to the pressure of $20 \mathrm{mmHg}$. On the first day, it was initially measured the intra-cuff pressure with the patient supine with the head at $30^{\circ}$, followed by the lateral decubitus (back to the ventilator) and the lateral position facing the ventilator. From the second to the fifth day, the pressure was checked only in the supine position with the head at $30^{\circ}$. For data analysis, both independent and dependent Student $t$ test were used $(p<0.05)$.

Results: In five consecutive days of verification of the intra-cuff pressure in the supine position we have observed that pressures were slightly decreased compared to the reference value, where the third day showed the lower pressure $(16.4 \pm 15.1 \mathrm{mmHg})$. When performing position changes it was noted that the intra-cuff pressure showed variations up or below $20 \mathrm{mmHg}$, that when analyzing the differences between the mean pressures, showed statistically significant results. The lateral decubitus with the back to the ventilator presented with pressure values within the reference frame $(20.3 \mathrm{mmHg})$, although a decrease was found in the decubitus facing the ventilator (18.1 \pm 4.8$)$.

Conclusion: In conclusion, the mean intra-cuff pressures in five consecutive days were decreased. Only on the fifth day pressures remained at normal values and the position changes significantly influenced the variation of this pressure.

Keywords: hospital physical therapy service; intensive care units; artificial respiration.

\section{Correspondência:}

DANIEL VICENTINI DE OLIVEIRA

Av. Londrina, 934, Apto. 1907, Torre A.

87050-730 Maringá, PR, Brasil

E-mail: d.vicentini@hotmail.com 


\section{INTRODUÇÃO}

Nas unidades de terapias intensivas (UTIs) grande parte dos pacientes acaba por necessitar da utilização da ventilação mecânica invasiva $(\mathrm{VMI})^{1,2}$, de forma a dar um suporte à função ventilatória comprometida ${ }^{3,4}$. No entanto, para sua aplicabilidade é necessária a utilização de via aérea artificial, cujas próteses são o tubo orotraqueal (TOT) ou a cânula de traqueostomia ${ }^{1,5}$.

A pressão gerada pelo balonete (cuff) sobre a mucosa traqueal pode sofrer variação e isso ocorre devido ao tamanho e as mudanças na posição do tudo orotraqueal e alterações no tônus da musculatura da traquéia ${ }^{1,6}$. A mucosa da árvore traqueobrônquica é muito delicada, sendo constituída de uma única camada epitelial de células ciliadas, cujo contato, mesmo que mínimo, determina lesões em curtos períodos ${ }^{2,3,7-12}$. Por causa destas especificidades é comum encontrar lesões devido à pressão produzida pelo balonete que são caracterizadas por edema celular, estenose traqueal, perda de cílios, hemorragia, ulceração, descamação do epitélio e necrose, isso quando a pressão de cuff está acima de $30 \mathrm{cmH}_{2} \mathrm{O}^{13}$. Quando a pressão está abaixo de $20 \mathrm{cmH}_{2} \mathrm{O}$ pode ocorrer aspiração do conteúdo gastroesofágico e orofaríngeo, o que deixa o paciente susceptível a quadros de infecções ${ }^{1}$.

Para evitar que isso ocorra é necessário observar o grau de pressão transmitido na parede da traquéia ${ }^{6}$, onde estudos apontam que os valores seguros para evitar essas lesões, são entre 20 a $30 \mathrm{cmH}_{2} \mathrm{O}^{13}$. Já para Camargo et al. ${ }^{3}$ esses valores devem estar situados entre 15 a $40 \mathrm{cmH}_{2} \mathrm{O}$. Outros autores ${ }^{14}$ utilizam a medida em milímetros de mercúrio $(\mathrm{mmHg})$, que recomendam 20 a $25 \mathrm{mmHg}$, ou 18 a $22 \mathrm{mmHg}^{15}$. Estes valores são baseados na pressão de perfusão sanguínea da mucosa traqueal, podendo ser de $20 \mathrm{mmHg}^{14}$, ou situando-se entre $20-30 \mathrm{cmH}_{2} \mathrm{O}$ ou $25-30 \mathrm{mmHg}^{16}$.

Contudo, aponta-se que os valores fisiológicos para um bom fluxo capilar é entre 20 a $30 \mathrm{mmHg}$. Assim, é de fundamental importância que a pressão do balonete seja mantida abaixo da pressão de perfusão da mucosa traqueal ${ }^{14}$ e suficiente para vedar a traquéia de forma a não permitir o escape de ar durante a ventilação ${ }^{13}$.

Além disso, com o uso dessas próteses, o paciente fica sujeito a possíveis contaminações de microorganismos que pode ocorrer devido a perda de esterelidade, seja por falta de higienização, não lavagem das mãos e a não troca de luvas entre os atendimentos, e outros fatores. Esses microorganismos juntamente com a secreção acumulada no sistema respiratório, ao entrar em contato com os pulmões podem gerar um quadro de pneumonia ${ }^{17}$. Portanto, para evitar quadros de broncoaspiração e lesões traqueais faz-se necessário a verificação da pressão intra-cuff de forma rotineira ${ }^{1}$, porém Camargo et al. ${ }^{3}$ relata que a mensuração dessa pressão tem sido negligenciada por profissionais da área hospitalar. Assim, este estudo teve por objetivo verificar a pressão intra-cuff de pacientes sob ventilação mecânica e a relação com a mudança de decúbito.

\section{MATERIAIS E MÉTODOS}

Trata-se de um estudo transversal, aprovado Comitê de ética e pesquisa do Centro Universitário Cesumar (UNICESUMAR) por meio do parecer $\mathrm{n}$ o $001 / 2012$, realizado no período de março a agosto de 2012.

Como critério de inclusão os pacientes deveriam estar sob ventilação mecânica invasiva com tubo orotraqueal e de ambos os sexos. Os critérios de exclusão foram pacientes menores de 18 anos e aqueles pacientes com o diagnóstico fechado de morte encefálica ou suspeita da mesma além de impossibilidade de mudança de decúbito. A coleta dos dados foi realizada após o familiar ou responsável pelo paciente assinar o Termo de Consentimento Livre e Esclarecido (TCLE). A amostra foi composta por 80 pacientes de ambos os sexos.

Para a verificação da pressão intra-cuff foi adaptado um cufômetro através de um manômetro de um esfigmomanômetro (Missuri ${ }^{\circledR}$ ), no qual a pressão do balonete foi mensurada em mmHg e a mesma não deveria ficar abaixo de $20 \mathrm{mmHg}$ e nem acima de $30 \mathrm{mmHg}^{13}$. Na realização dos ajustes da pressão foi padronizado $20 \mathrm{mmHg}$ devido a pressão de perfusão da mucosa traqueal, porém em situações que fosse observado escape de ar após o ajuste, a pressão seria ajustada até que houvesse plena vedação do TOT, e se houvesse necessidade de altas pressões, ou seja acima de $30 \mathrm{mmHg}$, o médico plantonista era avisado e a troca da via aérea era realizada.

Através de uma ficha pré-elaborada foram coletadas informações sobre idade, sexo, diagnóstico e pressão intracuff. As pressões intra-cuff foram verificadas em cinco dias consecutivos de forma a levantar se estas estavam adequadamente ajustadas e, somente no primeiro dia foi realizada a verificação durante a mudança de decúbito, de forma a buscar saber se este procedimento influenciaria nas pressões.

No primeiro dia, inicialmente foi mensurada e anotada a pressão intra-cuff com o paciente em decúbito dorsal (DD) com cabeceira a $30^{\circ}$ e se necessário realizado o ajuste da mesma. Após este procedimento o paciente foi posicionado em decúbito lateral de costa para o ventilador (DLCV) e realizado a mensuração da pressão intra-cuff. Em situação de alteração da pressão acima ou abaixo do valor padronizado era feito o ajuste em $20 \mathrm{mmHg}$ e na sequência posiciona- 
va-se em decúbito lateral de frente ao ventilador (DLFV) e realizava novamente a mensuração da mesma. Do segundo ao quinto dia a mensuração da pressão intra-cuff foi realizada com o paciente em DD com cabeceira a $30^{\circ}$ e se necessário realizado o ajuste da mesma. Para o ajuste das pressões foi utilizada uma seringa de $10 \mathrm{ml}$, lembrando que para cada paciente era utilizado um par de luvas e realizada a lavagem das mãos para não haver via de contaminação.

A análise dos dados foi realizada no software SPSS 22.0, mediante uma abordagem de estatística descritiva e inferencial. Na abordagem descritiva foi realizada a distribuição das frequências absolutas e relativas para as variáveis categóricas. Para as variáveis numéricas, inicialmente foi verificada a normalidade dos dados por meio do teste Kolmogorov-Smirnov. Como os dados apresentaram distribuição normal, foi utilizado o teste $t$ de Student independente para comparação entre grupos e o teste $t$ de Student dependente para comparação entre as posições de decúbito (DLFV e DLCV), utilizando média ( $\overline{\mathrm{x}}$ ) e desvio-padrão $(\mathrm{dp})$ para caracterização dos resultados. A significância adotada foi de $p<0,05$.

\section{RESULTADOS}

No período de estudo foi verificado as pressões intra-cuff de 80 pacientes, apresentando uma maior prevalência do sexo masculino (72,5\%) quando comparado com o feminino $(27,5 \%)$. A média de idade desses pacientes foi de $61,6 \pm 15,6$ anos. O diagnóstico mais frequente entre os pacientes foi: acidente vascular encefálico (AVE) (22,5\%), seguido de sepse $(12,5 \%)$, pneumonia $(11,25 \%)$ (traumatismo crânio encefálico (TCE) (10\%), insuficiência cardíaca congestiva (ICC) $(10 \%)$ e outros (33,75\%).

Ao analisar a Tabela 1, observa-se que as médias das pressões intra-cuff em cinco dias consecutivos apresentaramse diminuídas, principalmente no terceiro dia, e, notandose que apenas o quinto dia as pressões mantiveram-se em valores considerados normais ( $>20 \mathrm{e}<30 \mathrm{mmHg}$ ). Contudo, quando se observa o desvio padrão nota-se que sua variação foi importante no decorrer da semana, principalmente no quinto dia.

Ao comparar a pressão intra-cuff entre as duas posições de decúbito (Tabela 2), verificou-se que na posição de DLFV $(18,1 \pm 4,8)$ houve menor variação $(p=0,001)$ de pressão em comparação à posição de DLCV $(20,3 \pm 6,5)$, entretanto, ressalta-se que em ambas as posições a pressão se manteve próxima aos níveis mínimos de referência.

Ao comparar a pressão intra-cuff acima ou abaixo de $20 \mathrm{mmHg}$ após as mudanças de decúbito (Tabela 3), verificou-se diferença significativa tanto no $\operatorname{DLCV}(p=0,001)$ quanto no DLFV $(p=0,014)$. Ressalta-se que em ambas as posições os sujeitos com menos de $20 \mathrm{mmHg}$ de pressão intra-cuff apresentaram menor variação e com valores abaixo da referência.

Nota-se na Tabela 4 que, não houve diferença significativa na variação da pressão intra-cuff em função da faixa etária $(p>0,05)$, indicando que a idade não demonstrou ser uma variável interveniente na variação após mudança de decúbito.

Tabela 1. Média e desvio padrão da verificação da pressão de cuff no decúbito dorsal realizada durante 5 dias consecutivos.

\begin{tabular}{|cc|}
\hline Decúbito dorsal & $\begin{array}{c}\text { Pressão intra-cuff } \\
\overline{\mathbf{x}} \pm \mathbf{d p}\end{array}$ \\
\hline 1ㅇ dia & $18,1 \pm 16,3$ \\
20 dia & $18,4 \pm 18,6$ \\
3ㅇ dia & $16,4 \pm 15,1$ \\
4ㅇ dia & $17,8 \pm 16,9$ \\
5o dia & $25,4 \pm 24,5$ \\
\hline
\end{tabular}

$\overline{\mathrm{x}}$ : média; dp: desvio padrão.

Tabela 2. Comparação da pressão intra-cuff nas diferentes posições de decúbito.

\begin{tabular}{cccc}
\hline \multirow{2}{*}{ Variável } & \multicolumn{2}{c}{ Posições de Decúbito } & \\
\cline { 2 - 3 } & DLCV & DLFV & $\boldsymbol{p}$ \\
\hline \multirow{2}{*}{ Pressão intra-cuff } & $20,3 \pm 6,5$ & $18,1 \pm 4,8$ & $0,001^{*}$ \\
\hline
\end{tabular}

* Diferença significativa $(p<0,05)$. Teste $t$ de Student dependente.

$\bar{x}$ : média; dp: desvio padrão.

Tabela 3. Comparação da pressão intra-cuff acima ou abaixo de 20 mmHg após mudanças de decúbito.

\begin{tabular}{lccc}
\hline \multirow{2}{*}{ Decúbito } & \multicolumn{2}{c}{ Pressão intra-cuff $(\mathbf{m m H g})$} & \\
\cline { 2 - 3 } & $\begin{array}{c}\text { Menos de 20 (n=47) } \\
\overline{\mathrm{X}} \pm \mathbf{d} \mathbf{p}\end{array}$ & $\begin{array}{c}\mathbf{2 0} \text { ou mais }(\mathbf{n}=\mathbf{3 3}) \\
\overline{\mathrm{X}} \pm \mathbf{d p}\end{array}$ & $\boldsymbol{p}$ \\
\hline DLCV & $17,6 \pm 5,8$ & $24,3 \pm 5,4$ & $0,001^{*}$ \\
DLFV & $17,0 \pm 4,5$ & $25,5 \pm 4,7$ & $0,014^{*}$ \\
\hline
\end{tabular}

* Diferença significativa $(p<0,05)$. Teste $t$ de Student independente.

$\overline{\mathrm{X}}$ : média; dp: desvio padrão.

Tabela 4. Comparação da pressão intra-cuff nas duas posições de decúbito em função da idade.

\begin{tabular}{lccc}
\hline \multirow{2}{*}{ Decúbito } & \multicolumn{3}{c}{ Idade } \\
\cline { 2 - 3 } & $\begin{array}{c}\text { Menos de } \mathbf{6 0} \text { anos } \\
(\mathbf{n}=\mathbf{3 3}) \\
\overline{\mathrm{x}} \pm \mathbf{d} \mathbf{p}\end{array}$ & $\begin{array}{c}\text { Mais de } \mathbf{6 0} \text { anos } \\
(\mathbf{n}=\mathbf{4 7 )}\end{array}$ & $\boldsymbol{p}$ \\
$\overline{\mathrm{x}} \pm \mathbf{d p}$ & \\
\hline DLCV & $20,2 \pm 6,6$ & $20,4 \pm 6,5$ & 0,710 \\
DLFV & $18,4 \pm 4,9$ & $18,7 \pm 4,9$ & 0,254 \\
\hline
\end{tabular}

* Diferença significativa $(p<0,05)$. Teste $t$ de Student independente.

$\overline{\mathrm{x}}$ : média; dp: desvio padrão. 


\section{DISCUSSÃO}

Com base nos dados levantados neste estudo, observouse que o perfil dos pacientes na UTI e que necessitaram de VMI era de indivíduos dentro da faixa etária idosa, o que vai de encontro com outros estudos ${ }^{18,19}$. A população está envelhecendo e com isso o número de pessoas com doenças crônico-degenerativas vêm aumentando e desencadeando agravos à saúde com consequente necessidade de intervenções que requerem internação em UTI ${ }^{20}$.

No que tange esse perfil atualmente, justificaria o AVE, como o mais frequente motivo de admissão na amostra na UTI da presente pesquisa, que corrobora com o estudo de Gonçalves \& Padilha ${ }^{19}$ e Kunigk \& Chehter ${ }^{21}$ que apontaram frequência semelhante para este tipo de comprometimento $(30,4 \%)$.

A sepse refletiu em 12,5\% entre os motivos de internação no presente estudo, o que perto do estudo de Feijó e colaboradores ${ }^{18}$ mostrou-se muito inferior $(61,3 \%)$, porém é importante lembrar que a incidência de doenças infecciosas aumenta com a idade, e é responsável por um terço do óbito em pacientes acima de 65 anos e com uma taxa de mortalidade de 20 a $40 \%$.

Neste estudo utilizou a cabeceira a $30^{\circ}$, isto por ser um posicionamento que facilita o retorno venoso da cabeça e por ajudar na prevenção de pneumonia associada a $\mathrm{VMI}^{4}$ e para uma melhor padronização da metodologia, pois segundo Ono e colaboradores ${ }^{22}$, as pressões de cuff podem sofrer redução ao modificar as angulações da cabeceira do leito dos pacientes, principalmente conforme aumenta a angulação. Este dado nos mostra também que, devemos ter cautela ao elevar a cabeceira, devido a ocorrência de alterações na pressão de cuff.

Estudos apontam que a rotina da verificação da pressão de cuff, de forma a mantê-las adequadas, é negligenciada pelos profissionais que trabalham na área hospitalar ${ }^{3}$, e isto pode repercutir em momentos com altas ou baixas pressões de cuff. Esta última situação pode ser observada em quatro dias consecutivos em nossa amostra, fato que expõe o paciente a uma ventilação pulmonar inadequada por falta de vedação, e consequente broncoaspiração de secreções e pneumonia associadas à ventilação mecânica ${ }^{6,9,16}$. Contudo, há estudo que apontam alterações da pressão intra-cuff acima de $30 \mathrm{mmHg}^{1}$ e que após duas horas do contato do cuff com estas pressões elevadas inicia-se processo lesivo com consequente sequelas pós-extubação ${ }^{3}$.

Ao realizar a mudança de decúbito no paciente observouse que as pressões intra-cuff podem variar acima ou abaixo de $20 \mathrm{mmHg}$ independente do posicionamento. Quando o paciente foi posicionado em DLCV a média mostrou-se dentro dos valores esperados $(20,3 \mathrm{mmHg})$, diferente do DLFV que apresentou-se abaixo do referencial $(18,1 \mathrm{mmHg})$, porém o DLCV apresentou uma maior variação do desvio padrão $( \pm 6,5)$ quando comparado com o DLFV $( \pm 4,8)$. Segundo Godoy et al..$^{15}$ a variação da pressão ocorre em virtude da compressão ou descompressão do tubo endotraqueal sobre o balonete, que são causadas por movimentos e mudanças na posição do circuito do ventilador mecânico, porém o posicionamento em DLCV as variações das pressões são maiores em virtude de ser atribuído uma maior tensão no circuito de ar do ventilador.

Alguns fatores podem induzir a lesões no trato respiratório de indivíduos sob ventilação mecânica. Dentre eles, se destacam: umidificação inadequada da via aérea, alta fração de oxigênio inspirado, insuficiente aquecimento dos gases administrados, frequente sucção traqueal, prolongada entubação endotraqueal e valores inadequados de pressão de cuff ${ }^{23-27}$. Alguns autores sugerem que, entre esses fatores citados anteriormente, valores inadequados de pressão de cuff, ocorrendo uma variação, que isso é de grande valia na origem das lesões pós-entubação diagnosticadas no trato respiratório. Essas variações na pressão de cuff estão sujeitas a danificações na parede traqueal, bem como na facilitação de aspiração de conteúdo da orofaringe, levando a pneumonia nosocomia| ${ }^{23-25}$. No entanto, se a pressão de cuff ficar abaixo de $10 \mathrm{mmHg}$, microaspirações de conteúdo orofaríngeo podem ocorrer. O corte imediatamente acima do balonete coleta secreções orofaríngeas e das vias aéreas superiores, sendo a fonte de material para microaspirações se o balonete de ar perder a pressão ${ }^{28,29}$.

Alguns estudos relatam várias medidas de prevenção que poderiam ser tomadas para evitar dano ao sistema respiratório causado pela presença do tubo endotraqueal ou pela variação na pressão de cuff ${ }^{30}$. Dentre essas medidas, estão o controle regular e periódico da pressão de cuff, uso de tubos endotraqueais com adequado diâmetro, uso de uma válvula reguladorea de pressão de cuff no tubo piloto, uso de tubo endotraqueais com balonete de baixa pressão e alto volume e contínua aspiração do conteúdo orofaríngeo ${ }^{30}$.

Embora no início da década de 70 que os tubos endotraqueais com baolete de alto volume e baixa pressão, tenha reduzido a frequência das lesões traqueais pósentubação, este tipo de balonete, quando inflado com grandes volumes, pode alcançar pressões que após 2 a 4 horas danifica os tecidos ${ }^{31}$. Quando a pressão de cuff permanece entre os padrões de normalidade, os danos à parede tendem a ser reduzidos ou evitados. Mas após 2 a 12 horas de uma pressão de aproximadamente $20 \mathrm{mmHg}$, pode ocorrer um processo inflamatório no local em que o balonete está diretamente em contato. Sendo assim, 
a gravidade do processo é proporcional à duração da entubação ${ }^{24}$.

No entanto, as consequências da restrição prolongada no leito oriundo das mais diversas patologias que acometem os pacientes que necessitam de cuidados de terapia intensiva requerem que haja uma equipe altamente treinada e especializada de Médicos, Enfermeiros e Fisioterapeutas para que venha propiciarem as devidas mudanças de decúbito e a mobilização destes pacientes acamados, a fim de evitar lesões cutâneas e ulceras de pressão ${ }^{15}$, além do desmame rápido, pois estudos apontam que pacientes com 12 a 48 horas com tubo em contato com a mucosa já podem desenvolver lesões ${ }^{32}$. Assim, isto reforça a importância do treinamento das equipes de profissionais das UTIs, bem como a implantação de rotina para a verificação do cuff todas as vezes que o paciente for mobilizado, de forma a trazer benefícios a sua saúde.

Dentre as limitações do presente estudo, destacam-se a ausência de dados mais específicos de caracterização da amostra, pequeno tamanho amostral e a não homogeneidade da amostra tanto em relação à faixa etário e ao diagnóstico.

Conclui-se que as médias das pressões intra-cuff em cinco dias consecutivos apresentaram-se diminuídas, apenas no quinto dia as pressões mantiveram-se em valores considerados normais e que a mudança de decúbito influenciou significativamente na alteração desta pressão.

\section{REFERÊNCIAS}

1. Weiss V, Dornelas BR, Aragão GN, Silva JVM, Bezerra FS, Rocha Júnior AM. Avaliação das pressões intra-cuff em pacientes na unidade de terapia intensiva e sua relação com casos de pneumonias nasocomial. Saúde Pesq. 2010;3(3):315-20.

2. Rosa FK, Roese CA, Savi A, Dias AS, Monteiro MB. Comportamento da Mecânica pulmonar após a aplicação de protocolo de fisioterapia respiratória e aspiração traqueal em pacientes com ventilação mecânica invasiva. Rev Bras Ter Intensiva. 2007;19(2):170-5. https://doi.org/10.1590/S0103-507X2007000200005

3. Camargo MF, Andrade APAm Cardoso FPFM, Melo MHO. Análise das pressões intracuff em pacientes em terapia intensiva. Rev Assoc Med Bras. 2006;52(6):405-8. https://doi.org/10.1590/S010442302006000600019

4. Jerre G, Silva TJ, Beraldo MA, Gastaldo A, Kondo C, Leme F, Guimarães F, Forti Junior G, Lucato JJJ, Tucci MR, Vega JM, Okamoto VN. Fisioterapia no paciente sob ventilação mecânica. J Bras Pneumol.2007;33(2):142-50. https://doi.org/10.1590/S180637132007000800010

5. Pe-a ELC, Gregori WM, Piccicici Filho L, Vieira JE, Mathias LAST. Determinação de volumes e pressões de balonetes de tubos traqueais insuflados com ar ambiente ou óxido nitroso. Rev Bras Anestesiol. 2004;54(3):335-42. https://doi.org/10.1590/S003470942004000300007
6. Taveira SSVL, Ribeiro R, Silvino SE, Pinto WM. Conhecimento teórico-prático da equipe assistente sobre manejo e pressão do balonete endotraqueal. Conscientiae Saúde. 2009;8(1): 25-34.

7. Aranha AGA, Forte V, Perfeito JAJ, Villaça LE, Imaeda CJ, Juliano Y. Estudo das pressões no interior dos balonetes de tubos traqueais. Rev Bras Anestesiol. 2003;53(6):728-36. https://doi.org/10.1590/ S0034-70942003000600004

8. Castilho EC, Braz JR, Catâneo AJM, Martins RHG, Gregório EA, Monteiro ER. Efeitos da pressão limite $(25 \mathrm{cmH} 2 \mathrm{O})$ e mínima de "selo" do balonete de tubos traqueais sobre a mucosa traqueal do cão. Rev Bras Anestesiol. 2003;53(6):743-55. https://doi. org/10.1590/S0034-70942003000600006

9. Mendes FF, Hintz L, Bredemeier Neto F. Volume e pressão do balonete do tubo traqueal para oclusão da traquéia. Rev Bras Anestesiol.1996;46(2):103-6.

10. Mota LAA, Carvalho GB, Brito VA. Complicações laringeas por intubação orotraqueal: Revisão da literatura. Int. Arch Otorhinolaryngol. 2012;16(2):236-45. https://doi.org/10.7162/ s1809-48722012000200014

11. Martins RHG, Dias NH, Braz JRC, Castilho EC. Complicações das vias aéreas relacionadas à intubação endotraqueal. Rev Bras Otorrinolaringol. 2004;70(5):671-7. https://doi.org/10.1590/S003472992004000500015

12. Stwart SL, Secrest JA, Norwood BR, Zachary R. Comparison of endotracheal tube cuff pressures using estimation techniques and direct intracuff measurement. J Am Assoc Nurse Anesth. 2003; 71(6):443-7.

13. Penitenti RM, Cilches JIG, Oliveira JSM, Mizohata GG, Correa DI, Alonso TRMB, Mathiazzi IC, Testa RS. Controle da pressão do cuff na unidade de terapia intensiva: efeitos do treinamento. Rev Bras Ter Intensiva. 2010;22(2):192-5. https://doi.org/10.1590/S0103507X2010000200014

14. Cardoso GS, Guimarães HP, Lopes RD, Leal PHR, Souza F, Guedes CJ, Senna APR, Machado FR, Amaral JLG. Controle da pressão do balonete de cânulas traqueais: estudo prospectivo em unidade de terapia intensiva geral. Rev Bras Ter Intensiva. 2005;17(3): 185-7.

15. Godoy ACF, Vieira ACF, Capitani EM. Alteração da pressão intra-cuff do tubo endotraqueal após mudança da posição em pacientes sob ventilação mecânica. J Bras Pneumol. 2008;34(2):294-7. https://doi. org/10.1590/S1806-37132008000500008

16. Juliano SRR, Juliano MCR, Cividanes JP, Houly JGS, Gebara OCE, Cividanes GVL, Catão EC. Medidas dos níveis de pressão do balonete em unidade de terapia intensiva: considerações sobre os benefícios do treinamento. Rev Bras Ter Intensiva. 2007;19(3): 317-21. https://doi.org/10.1590/S0103-507X2007000300008

17. Teixeira PJZ, Hertz FT, Cruz DB, Caraver F, Hallal RC, Moreira JS. Ventilator-associated pneumonia: impact of bacterial multidrug resistance on morbidity and mortality. J Bras Pneumol. 2004;30(3):540-8

18. Feijo CAR, Bezerra ISAM, Peixoto Junior AA, Meneses FA. Morbimortalidade do idoso internado na unidade de terapia intensiva de Hospital Universitário de Fortaleza. Rev Bras Ter Intensiva. 2006;8(3):263-7. https://doi.org/10.1590/S0103507X2006000300008 
19. Gonçalves LA, Padrilha KG. Fatores associados à carga de trabalho de enfermagem em Unidade de Terapia Intensiva. Rev Esc Enferm USP. 2007;41(4):645-52. https://doi.org/10.1590/S008062342007000400015

20. Costa SRD, Castro EDB, Acioli S. Capacidade de autocuidade de adultos e idosos hospitalizados: implicações para o cuidado de enfermagem. Rev Min Enferm. 2013;17(1):193-207.

21. Kunigki MR, Chehter E. Disfagia orofaríngea em pacientes submetidos à entubação orotraqueal. Rev Soc Bras Fonoaudiol. 2007;12(4):287-91. https://doi.org/10.1590/S1516-8034200 7000400006

22. Ono FC, Andrade APA, Cardoso FPF, Melo MHO, Souza RN, Silva GHC, Vieira BEM. Análise das pressões de balonetes em diferentes angulações da cabeceira do leito dos pacientes internados em unidade de terapia intensiva. Rev Bras Ter Intensiva. 2008;20(3): 220-5. https://doi.org/10.1590/S0103-507X2008000300003

23. Berlauk JF. Prolonged endotracheal intubation vs. tracheostomy. Crit Care Med. 1986;14(8):742-5. https://doi.org/10.1097/00003246198608000-00017

24. Sarper A, Ayten A, Eser I, Ozbudak O, Demircan A. Tracheal stenosis aftertracheostomy or intubation: review with special regard to cause and management. Tex Heart Inst J. 2005;32(2):154-8.

25. Fan CM, Ko PC, Tsai KC, Chiang WC, Chang YC, Chen WJ, Yuan A. Tracheal rupture complicating emergent endotracheal intubation. Am J Emerg Med. 2004;22(4):289-93. https://doi.org/10.1016/j. ajem.2004.04.012
26. Badenhorst $\mathrm{CH}$. Changes in tracheal cuff pressure during respiratory support. Crit Care Med. 1987;15(4):300-2. https://doi. org/10.1097/00003246-198704000-00003

27. Nseir S, Di Pompeo C, Pronnier P, Beague $S$, Onimus T, Saulnier F, Grandbastien B, Mathieu D, Delvallez-Roussel M, Durocher A. Nosocomial tracheobronchitis in mechanically ventilated patients: incidence, aetiology and outcome. Eur Respir J. 2002;20(6):1483-9. https://doi.org/10.1183/09031936.02.00012902

28. Bernhard WN, Yost L, Joynes D, Cothalis S, Turndorf H. Intracuff pressures in endotracheal and tracheostomy tubes. Related cuff physical characteristics. Chest. 1985;87(6):720-5. https://doi. org/10.1378/chest.87.6.720

29. Wunderink RG. Nosocomial pneumonia, including ventilatorassociated pneumonia. Proc Am Thorac Soc. 2005;2(5):440-4. https://doi.org/10.1513/pats.2005080-83JS

30. Farré R, Rotger M, Ferre M, Torres A, Navajas D. Automatic regulation of the cuff pressure in endotracheally-intubated patients. Eur Respir J. 2002;20(4):1010-3. https://doi.org/10.1183/09031936.02.02692001

31. Klainer AS, Turndorf H, Wu WH, Maewal H, Allender P. Surface alterations due to endotracheal intubation. Am J Med. 1975; 58(5):674-83. https://doi.org/10.1016/0002-9343(75)90504-5

32. Cordeiro AMG, Shin SH, Fernandes ICOF, Bousso A, Troster EJ. Incidência e características endoscópias de lesões das vias aéreas associadas a intubação traqueal em crianças. Rev Assoc Med Bras. 2004;50(1):87-92. https://doi.org/10.1590/S010442302004000100042 